\title{
The Sectored Antenna Array Indoor Positioning System with Neural Networks
}

\author{
Chih-Yung Chen ${ }^{1}$, Yu-Ju Chen ${ }^{2}$, Ya-Chen Weng ${ }^{1}$, Shen-Whan Chen ${ }^{3}$, Rey-Chue Hwang ${ }^{4}$, * \\ ${ }^{1}$ Department of Computer and Communication, Shu-Te University, Kaohsiung City, Taiwan \\ ${ }^{2}$ Department of Information Management, Cheng Shiu University, Kaohsiung City, Taiwan \\ ${ }^{3}$ Department of Communication Engineering, I-Shou University, Kaohsiung City, Taiwan \\ ${ }^{4}$ Department of Electrical Engineering, I-Shou University, Kaohsiung City, Taiwan
}

\section{Email address:}

mikechen@stu.edu.tw (Chih-Yung Chen), yjchen@csu.edu.tw (Yu-Ju Chen), s97115209@stu.edu.tw (Ya-Chen Weng), jasonchen@isu.edu.tw (Shen-Whan Chen),rchwang@isu.edu.tw (Rey-Chue Hwang)

${ }^{*}$ Corresponding author

\section{To cite this article:}

Chih-Yung Chen, Yu-Ju Chen, Ya-Chen Weng, Shen-Whan Chen, Rey-Chue Hwang. The Sectored Antenna Array Indoor Positioning System with Neural Networks. Automation, Control and Intelligent Systems. Vol. 4, No. 2, 2016, pp. 21-27. doi: 10.11648/j.acis.20160402.13

Received: February 22, 2016; Accepted: March 21, 2016; Published: March 25, 2016

\begin{abstract}
This paper presents a sectored antenna array indoor positioning system (IPS) with neural network (NN) technique. The hexagonal positioning station is composed of six printed-circuit board Yagi-Uda antennas and Zigbee modules. The values of received signal strength (RSS) sensed by wireless sensors were used to be the information for object's position estimation. Two NN models, including NN with back-propagation (BP) learning algorithm and probabilistic NN (PNN), were applied to perform the positioning work for a comparison. In the experiments, an $8 \times 8$ square meters indoor scene was performed and 288 points and 440 points were experimented in this area. The positioning results show that both NN models have the average error less than 0.7 meter. In other words, the proposed positioning system not only has the high positioning accuracy, but also has the potential in real application.
\end{abstract}

Keywords: Sectored Antenna, Indoor Positioning System (IPS), Neural Network (NN), Received Signal Strength (RSS)

\section{Introduction}

It is well known that IPS has become more and more popular in the object searching due to the rapid developments of wireless communication technique and personal network [1-3]. IPS is used to provide the location information of person and device. It is a system of location based service (LBS) through the integration of the wireless communication and information services. The object's accurate position could be determined by using such a service system. It has been used widely in the various applications, such as cargo management, patient monitoring, public guiding system, etc. Undoubtedly, IPS will certainly play a significant role for the smart life of human beings in the future.

Generally, the structure of IPS could be divided into two parts, i.e. the positioning algorithm and the sensing infrastructure. The positioning algorithm is the method of determining the object's location. So far, three algorithms, including triangulation, scene analysis and proximity, are mainly used for the object's position estimation [4-9]. The sensing infrastructure is related to the wireless communication technology used for IPS. Nowadays, the various wireless communication technologies such as wireless local area network (WLAN) [10-13], wireless sensor network (WSN) [14-15], radio frequency identification (RFID) [16-18], Bluetooth [19-20], Zigbee [21-22], etc. have been wildly used in the sensing technique of IPS. Each IPS has its advantage and limitation in accordance with the developed element's function. In which, many positioning computational algorithms used the values of RSS sensed from the known reference nodes to calculate the object's coordinate [23-24]. But, since the influences of external factors such as the obstacle of hindrance, the noise disturbance and the diffraction of electromagnetic wave, the positioning computational method is still a challenging topic in the research of IPS application. 
In recent years, due to the powerful learning and adaptive capabilities, NN technique has been employed into the positioning applications [25-31]. It is used to catch the nonlinear mapping between the coordinate of object and RSS signals. Through a training process, the well-trained NN model then can be used to estimate the object's location based on RSS measurements. In this research, two NN models, including NN with BP learning algorithm and PNN, were applied to perform the positioning work for a comparison. The detailed NN models will be described in the following section.

In order to improve the positioning accuracy, Cidronali et al [32] designed a new switched beam array antenna for wireless indoor positioning application. The antenna is intended to augment a wireless device operating as the coordinator or base station, and its design is suitable for installation on the ceiling of any large indoor space [33-34]. Similar to [32], this paper presents a novel indoor positioning scheme which is composed of array antennas and Zigbee modules. The information of signal angle and RSS are used to estimate the object's location. The whole paper is organized as follows. The proposed indoor positioning system is presented in Section 2. Section 3 describes the NN models for the positioning estimation. Section 4 presents the relevant experiments and results. At last, a conclusion is given in Section 5.

\section{The Proposed Indoor Positioning System}

In this research, Figure 1 shows the developed IPS module which consists of two parts. One part is the indoor positioning station and the other part is the embedded positioning device. The indoor positioning station is composed of a sectored antenna array, a microcontroller and six Zigbee modules. The sectored antenna array has six printed-circuit board (PCB) Yagi-Uda antennas with hexagonal arrangement which can provide 360 degrees coverage.

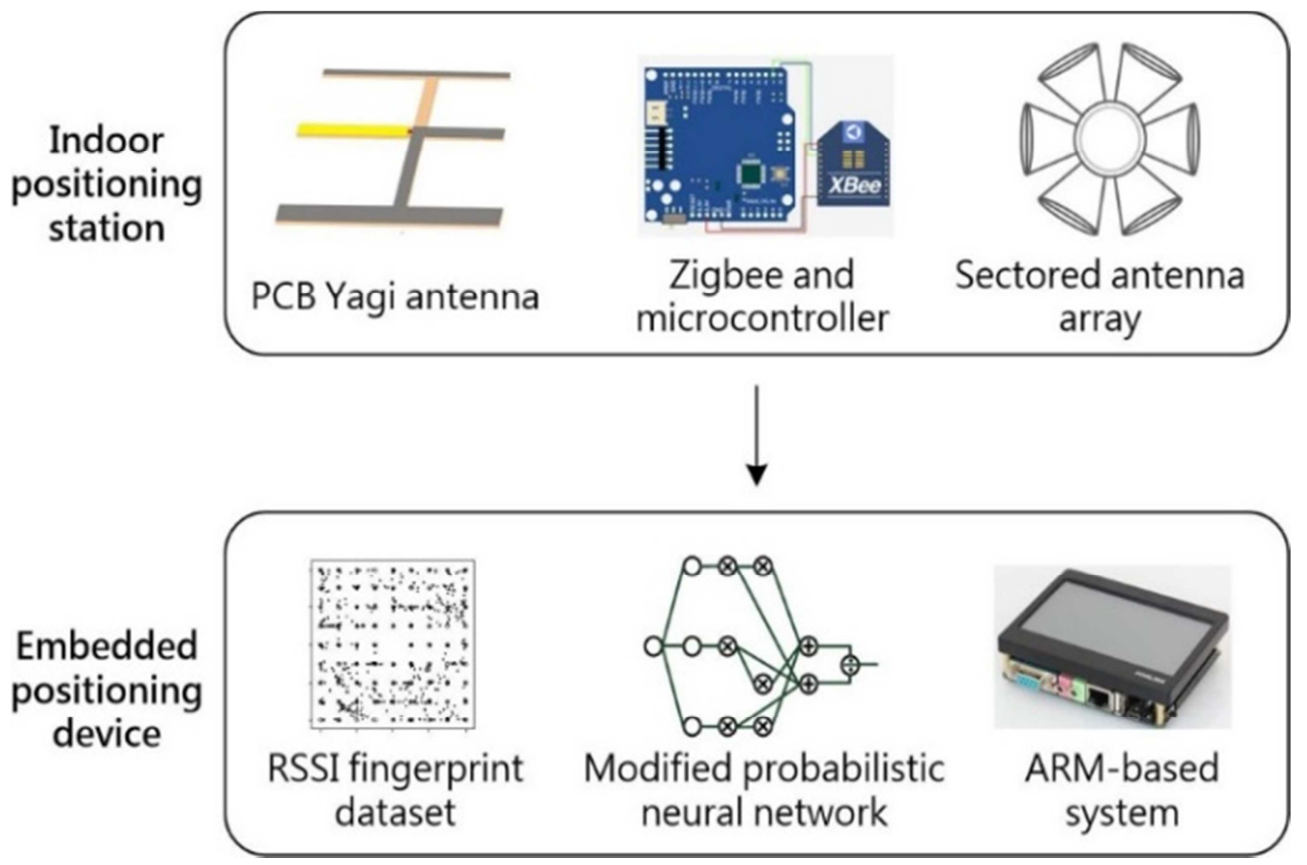

Figure 1. The proposed indoor positioning system.

Yagi-Uda antenna is one of the most successful radio frequency directional antenna designs. It has been used in a wide variety of applications that the antenna design needs gain and directivity [35]. Figure 2 shows the figures of Yagi-Uda antenna and its radiation pattern.
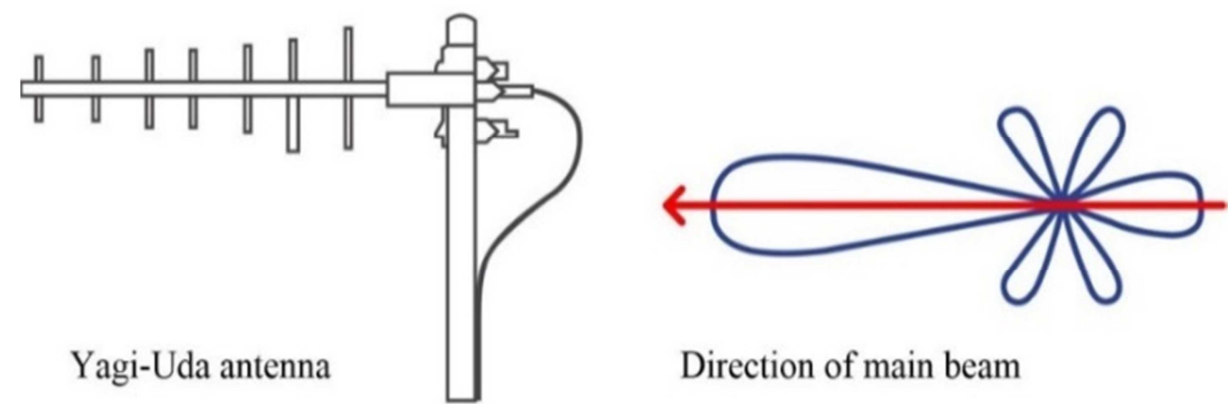

Direction of main beam

Figure 2. Yagi-Uda antenna and radiation pattern. 
The embedded positioning device is developed to perform the indoor positioning function. Through a training process, NN model could calculate the object's position by using RSS fingerprint dataset obtained from the station. Then, the positioning result could be displayed on the screen of ARM-based system.

The RSS based positioning technique estimates the position from the signals of RSS vector which can be obtained by wireless sensors. The radio propagation model with positioning algorithm is a common way to determine the distance between an object and the station. Its equation is generally expressed as

$$
R S S=-1 \cdot\left(10 \cdot n_{p} \cdot \log _{10} d+A\right)
$$

Where $n_{p}$ is the signal propagation constant, $d$ is the distance between object and sensor and $A$ is the object's RSS when the distance is 1 meter.

\section{NN and Modified PNN}

In our studies, traditional NN model with BP learning algorithm and modified PNN model were employed into the positioning works for a comparison. Two NN models are briefly described as follows.

\subsection{NN Model}

A three-layered feed-forward fully connected $\mathrm{NN}$ is used in the studies. The size of NN is 6-13-2 which means NN has 6 input nodes, 13 hidden nodes and 2 output nodes. It structure is shown in Figure 3. Two output nodes estimate the values of $x$ and $y$ axes of object's coordinate, respectively.

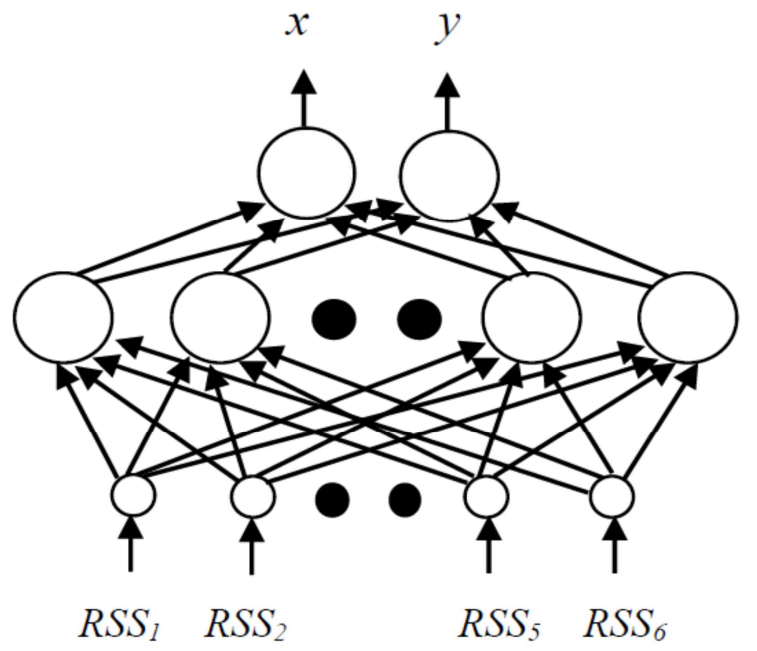

Figure 3. The NN structure.

The error back-propagation (BP) learning algorithm is adopted by NN model. The learning process is all training inputs are presented cyclically until all weights of NN are stabilized. The major steps of BP learning algorithm are summarized as follows [36-37].
Step 1: Initialize all weights, $\omega_{i j}$ to small random values (typically between -0.5 to 0.5 ).

Step 2: Present an input pattern and specify the desired output. Calculate output using the presents $\omega_{i j}$.

Step 3: Find the error term, $\delta$ for all nodes. If $D_{j}, O_{j}$ and $H_{j}$ denote the desired value of $j^{\text {th }}$ output node, the computed value of $j^{\text {th }}$ output node, and the computed value of $j^{\text {th }}$ hidden node, then the error terms of all nodes could be calculated by using the following equations.

The error of output layer node $j$ :

$$
\boldsymbol{\delta}_{j}=\left(D_{j}-O_{j}\right) O_{j}\left(1-O_{j}\right)
$$

The error of hidden layer node $j$ :

$$
\delta_{j}=H_{j}\left(1-H_{j}\right) \sum_{k} \omega_{j k} \delta_{k}
$$

where $k$ is over all nodes in the layer above node $j$.

Step 4: Adjust weights by

$$
\omega_{i j}(n+1)=\omega_{i j}(n)+\eta \delta_{j} u_{i}+\zeta\left(\omega_{i j}(n)-\omega_{i j}(n-1)\right)
$$

Where $(n+1),(n)$ and $(n-1)$ indicate the next, present, and previous iteration numbers, respectively. $u_{i}$ is the $i^{\text {th }}$ input connected with node $j . \eta$ is the learning rate. $\zeta$ is a momentum that effectively filters out high-frequency variations of the error surface.

Step 5: Present next input pattern and go back to step 2.

\subsection{Modified PNN}

The modified PNN was initialized by Zaknich [25]. In this research, PNN is applied to estimate the coordinate of object. The architecture of modified PNN is shown in Figure 4. It consists of one input layer, one pattern layer, one summing layer and one output layer. The algorithm of modified PNN is described as follows.

Let $\mathbf{C}$ be a set of class vectors i.e. IPS training data, which is given by

$$
\mathbf{C}=\left\{\left(c_{1}, y_{1}\right),\left(c_{2}, y_{2}\right) \ldots,\left(c_{m}, y_{m}\right)\right\}
$$

where $m$ is the number of class vectors. $c_{i}$ contains six RSS signals sensed by antenna and $y_{i}$ is the scalar output related to $c_{i}$. Here, the probability density function (PDF) of modified $\mathrm{PNN}$ is defined as

$$
\Phi\left(x, c_{i}, \sigma\right)=\exp \left(-\frac{\left(x-c_{i}\right)^{T}\left(x-c_{i}\right)}{2 \sigma^{2}}\right)
$$

where $\sigma$ is the smoothing parameter of Gaussian function, $x$ is the training vector for class $i$ in the input space. Thus, the output $\hat{y}$ i.e. the coordinate of object can be obtained by 


$$
\hat{y}(x)=\frac{\sum_{i=1}^{m} z_{i} y_{i} \Phi\left(x, c_{i}, \sigma\right)}{\sum_{i=1}^{m} z_{i} \Phi\left(x, c_{i}, \sigma\right)}
$$

where $z_{i}$ is the number of $x_{i}$ associated with $c_{i}$.

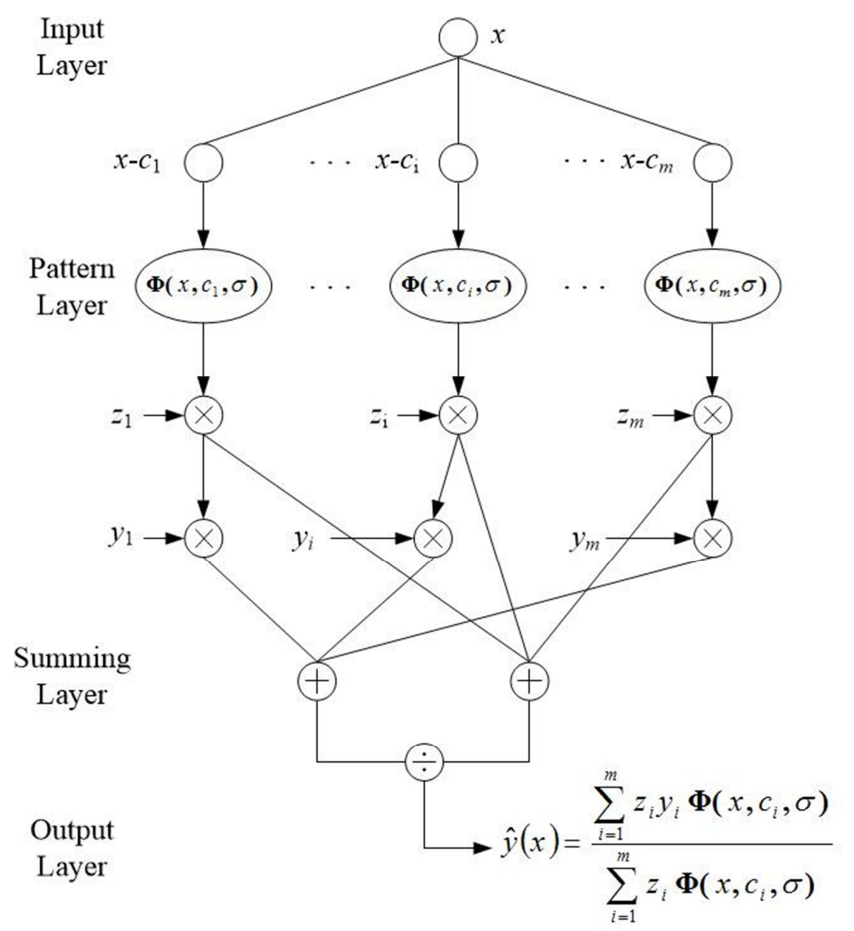

Figure 4. The architecture of $P N N$.

\section{Experiments and Results}

In this research, an $8 \times 8$ square meters indoor field as shown in Figure 5 is used for the experiments. In order to test the indoor positioning system developed, 288 and 440 positions (features) within the intervals of 0.5 meter and 0.4 meter were measured, respectively. The features collected by IPS station are RSS signals measured by wireless signal receiver. Figure 6 and Figure 7 present the illustrated figures for 288 and 440 test positions, respectively.

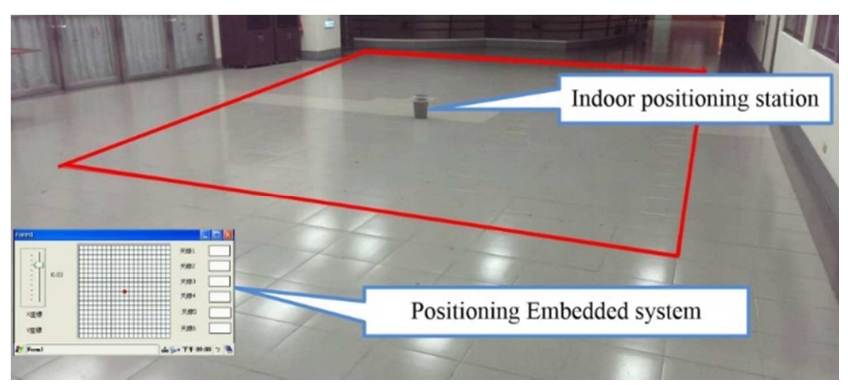

Figure 5. The indoor experimental field.

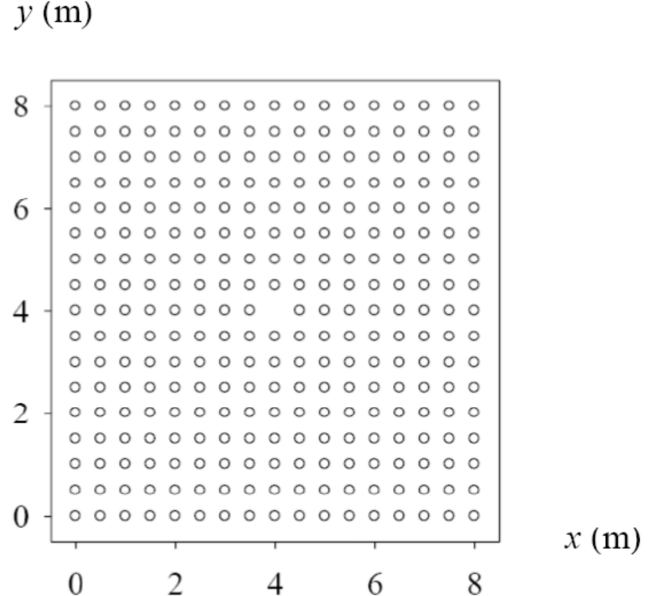

Figure 6. The illustrated figure for 288 positions.

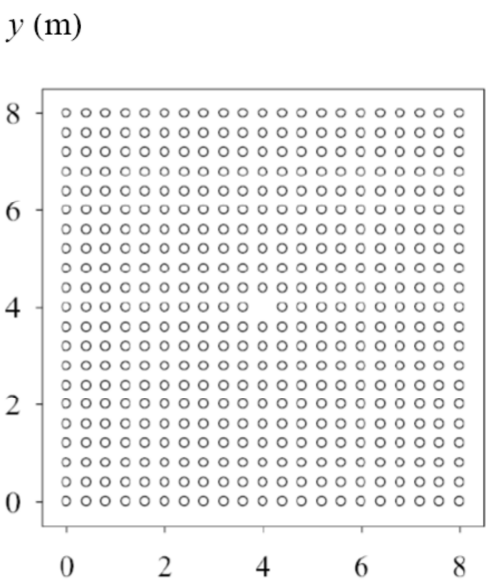

Figure 7. The illustrated figure for 440 positions.

\subsection{The Experiments by NN with BP Learning Algorithm}

Firstly, NN model with BP learning algorithm was used to do the object's position estimation. For 288 points experiment, two data groups were collected randomly, each group has 288 data sets and each set includes the information of point's coordinate $(\mathrm{x}, \mathrm{y})$ and six RSS sensed values $\left(\mathrm{RSS}_{1}, \mathrm{RSS}_{2}, \ldots, \mathrm{RSS}_{6}\right)$. First data group was used for NN's training and second data group was used for test. Table 1 lists the mean absolute errors (MAEs) of positional estimations by using 6-13-2 NN model with 10 different learning rates. From the results shown, the best performance is taken by NN model with 0.1 learning rate. The training MAE and test MAE are $48.5 \mathrm{~cm}$ and $52.09 \mathrm{~cm}$, respectively. Similarly, two data groups for 440 points were collected either. The same experiment was redone by 6-13-2 NN model. The positioning results is also shown in Table 1 . Again, the best performance is taken by NN model with 0.1 learning rate. The training MAE and test MAE are $62.11 \mathrm{~cm}$ and $63.80 \mathrm{~cm}$, respectively.

In this part of experiments, $\mathrm{NN}$ is viewed as the nonlinear regression model for performing a nonlinear input-output mapping. NN generates an approximate function to the training data. 
Table 1. The statistic errors of 288 and 440 positional estimations by NN.

\begin{tabular}{|c|c|c|c|c|}
\hline \multirow{2}{*}{$\begin{array}{l}\text { Learning } \\
\text { rate }\end{array}$} & \multicolumn{2}{|l|}{288 points } & \multicolumn{2}{|c|}{440 points } \\
\hline & $\begin{array}{l}\text { Training } \\
\text { MAE }\end{array}$ & Test MAE & $\begin{array}{l}\text { Training } \\
\text { MAE }\end{array}$ & Test MAE \\
\hline 0.1 & 48.50 & 52.09 & 62.11 & 63.80 \\
\hline 0.2 & 55.04 & 55.26 & 62.73 & 68.02 \\
\hline 0.3 & 59.26 & 56.88 & 65.83 & 69.63 \\
\hline 0.4 & 50.90 & 57.71 & 60.56 & 65.95 \\
\hline 0.5 & 57.22 & 58.41 & 63.55 & 70.36 \\
\hline 0.6 & 59.20 & 58.84 & 62.48 & 69.06 \\
\hline 0.7 & 60.64 & 59.60 & 64.58 & 71.39 \\
\hline 0.8 & 56.57 & 59.86 & 67.88 & 69.41 \\
\hline 0.9 & 58.19 & 61.32 & 63.89 & 67.92 \\
\hline 1.0 & 60.99 & 64.99 & 66.22 & 70.61 \\
\hline Avg. & 56.65 & 58.50 & 63.98 & 68.62 \\
\hline
\end{tabular}

\subsection{The Experiments by Modified PNN}

In the experiment by using PNN model, the same data groups were performed by modified PNN with 10 different $\sigma$ values. Table 2 presents the estimated positioning errors. From the results shown, PNN model with $\sigma=0.01$ has the best estimation. The estimated MAEs of 288 points and 440 points could reach to $1.96 \mathrm{~cm}$ and $1.72 \mathrm{~cm}$. Figure 8 and Figure 9 show the plots of 288 and 440 positional estimations. The symbols of circle and dot are actual and estimated coordinates of the experimental data.

Unlike previous NN's experiments, in this part of research, the modified PNN is viewed a classifier which is used to estimate the object's position in accordance with the features (RSS signals) sensed. Compare the results of Table 1 with Table 2, it is clearly found that the positioning accuracy performed by modified PNN is much better than NN with BP learning rule.

Table 2. The statistic errors of 288 and 440 positional estimations by modified PNN.

\begin{tabular}{lll}
\hline \multirow{\sigma}{*}{} & $\mathbf{2 8 8}$ points & $\mathbf{4 4 0}$ points \\
\cline { 2 - 3 } & MAE & MAE \\
\hline$\sigma=0.1$ & 109.88 & 108.97 \\
$\sigma=0.09$ & 95.77 & 95.97 \\
$\sigma=0.08$ & 82.00 & 83.40 \\
$\sigma=0.07$ & 68.82 & 71.15 \\
$\sigma=0.06$ & 55.82 & 58.70 \\
$\sigma=0.05$ & 42.33 & 45.20 \\
$\sigma=0.04$ & 28.08 & 29.77 \\
$\sigma=0.03$ & 13.76 & 14.45 \\
$\sigma=0.02$ & 4.26 & 4.49 \\
$\sigma=0.01$ & 1.96 & 1.72 \\
Avg. & 50.27 & 51.38 \\
\hline
\end{tabular}

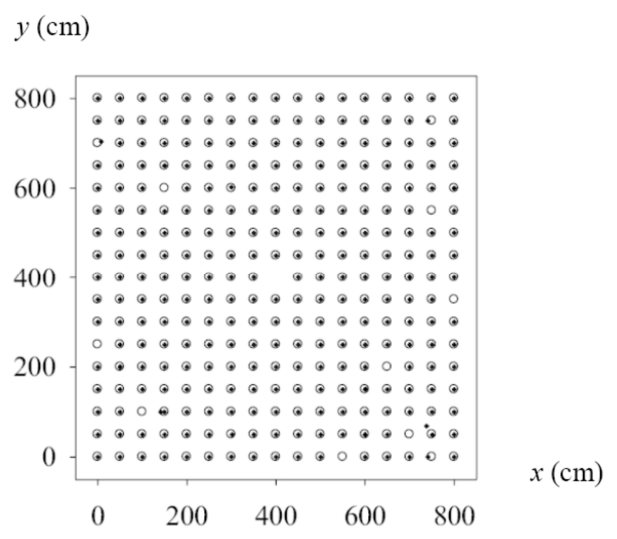

Figure 8. The plot of 288 positional estimations.

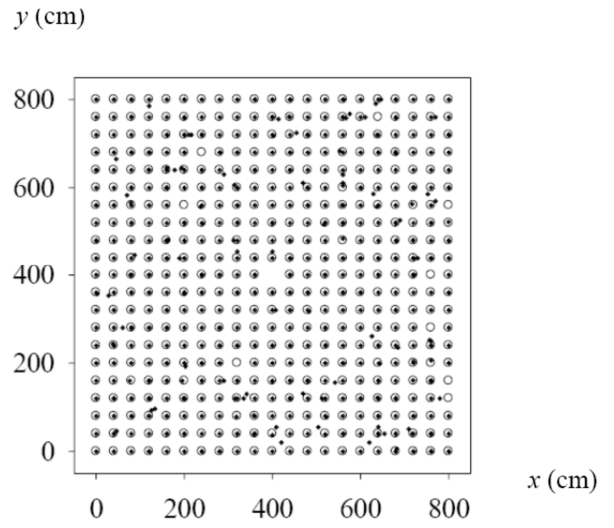

Figure 9. The plot of 440 positional estimations.

\section{Conclusion}

This paper presents a sectored antenna array indoor positioning system which structure includes the positioning algorithm and the sensing infrastructure. The infrastructure is composed of six printed-circuit board Yagi-Uda antennas and Zigbee modules which are used to generate and sense the RSS signals. Two NN models are the positioning methods to perform the object's position estimation according to the signals of RSS sensed. In our studies, the positioning accuracy performed by modified PNN model is much better than traditional NN. That means the classifier by modified PNN has the outperformance than the regression function generated by traditional $\mathrm{NN}$ in indoor positioning application. However, such a conclusion is given by the environment of RSS based positioning system must be stable and the signals of RSS have no serious problem caused by the effects of interference, diffraction or reflection. From the results shown in Table 1 and Table 2. It is able to be found that the estimated accuracies performed by modified PNN model are highly related to the values of $\sigma$. On the contrary, compare with the modified PNN model, the NN model with BP learning is more stable in performing the estimations. The variances of estimations are small. Thus, how to establish a more excellent and stable indoor positioning system is still the future work we will continue. 


\section{Acknowledgements}

This research was supported by the Ministry of Science and Technology, Taiwan, ROC under Contracts No. MOST-104-2221-E-366-004, No. MOST-104-2632-E-366-001 and No. MOST-103-222-E-214-050.

\section{References}

[1] Y. Y. Gu, A. Lo, I. Niemegeers, "A survey of indoor positioning systems for wireless personal networks," IEEE Communications Surveys \& Tutorials, vol. 11, no. 1, pp. 13-32, 2009.

[2] H. Liu, H. Darabi, P. Banerjee, J. Liu, "Survey of wireless indoor positioning techniques and systems," IEEE Trans. on Systems, Man, and Cybernetics, vol. 37, no. 6, pp. 1067-1077, 2007.

[3] G. W. Shi, Y. Ming, "Survey of indoor positioning systems based on ultra-wideband (UWB) technology," Lecture Notes in Electrical Engineering, Wireless Communications, Networking and Applications, Proceedings of WCNA 2014, Vol. 348, pp. 1269-1278, 2016.

[4] B. Kim, W. Bong, Y. C. Kim, "Indoor localization for Wi-Fi devices by cross-monitoring AP and weighted triangulation," In the Proceedings of IEEE Consumer Communications and Networking Conference (CCNC), NV, U.S.A., pp. 933-936, 2011.

[5] Y. Mo, Z. Z. Zhang, Y. Lu, G. Agha, "A novel technique for human traffic based radio map updating in Wi-Fi indoor positioning systems," KSII Transactions on Internet and Information Systems, vol. 9, no. 5, pp. 1881-1903, 2015.

[6] X. F. Jiang, C. J. Mike Liang, K. F. Chen, B. Zhang, J. Hsu, J. Liu, B. Cao, F. Zhao, "Design and evaluation of a wireless magnetic-based proximity detection platform for indoor applications," In the Proceedings of 11th ACM/IEEE International Conference on Information Processing in Sensor Networks (IPSN/SPOTS), Beijing, China, pp. 221-231, 2012.

[7] J. Hightower, G. Borriello, "Location sensing techniques," Technical Report UW CSE 2001-07-30, Department of Computer Science and Engineering, University of Washington, 2001.

[8] K. Kaemarungsi, P. Krishnamurthy, "Properties of indoor received signal strength for WLAN location fingerprinting," In the Proceedings of 1st Annual International Conference on Mobile and Ubiquitous Systems: Networking and Services (MobiQuitous '04), MA, USA, pp. 14-23, 2004.

[9] D. Focken, R. Stiefelhagen, "Towards vision-based 3-D people tracking in a smart room," In the Proceedings of 4th IEEE Intl Conference on Multimodal Interfaces, PA, USA, pp. 400-405, 2002.

[10] A. Kotanen, M. Hannikainen, H. Leppakoski, T. D. Hamalainen, "Positioning with IEEE 802.11b wireless LAN," In the Proceedings of 14th IEEE Proceedings on Personal, Indoor and Mobile Radio Communications, Beijing, China, pp. 2218-2222, 2003.

[11] Y. B. Xu, M. Zhou, L. Ma, "Hybrid FCM/ANN indoor location method in WLAN environment," In the Proceedings of IEEE
Youth Conference on Information, Computing and Telecommunications, Beijing, China, pp. 475-478, 2009.

[12] V. Honkavirta, T. Perala, S. Ali-Loytty, R. Piche, "A comparative survey of WLAN location fingerprinting methods," In the Proceedings of 6th Workshop on Positioning, Navigation and Communication (WPNC'09), Hannover, Germany, pp. 243-251, 2009.

[13] M. Y. Umair, K. V. Ramana, D. K. Yang, "An enhanced K-Nearest Neighbor algorithm for indoor positioning systems in a WLAN," 2014 IEEE Computers, Communications and Its Applications, pp. 19-23, January 20, 2014.

[14] K. F. S. Wong, I. W. Tsang, V. Cheung, S. H. G. Chan, J. T. Kwok, "Position estimation for wireless sensor networks," In the Proceedings of IEEE Global Telecommunications Conference, MO, USA, pp. 2772-2776, 2005.

[15] S. Aomumpai, K. Kondee, C. Prommak, K. Kaemarungsi, "Optimal placement of reference nodes for wireless indoor positioning systems," 11th International Conference on Electrical Engineering, Electronics, Computer, Telecommunications and Information Technology. Paper no. 6839894, 2014

[16] P. Bahl V. N. Padmanabhan, "RADAR: An in-building RF-based user location and tracking system," In the Proceedings of INFOCOM 2000, Nineteenth Annual Joint Conference of the IEEE Computer and Communications Societies, Tel Aviv, Israel, pp. 775-784, 2000.

[17] H. D. Chon, S. Jun, H. Jung, S. W. An, "Using RFID for accurate positioning," Journal of Global Positioning Systems, vol. 3, pp. 32-39. 2004.

[18] H. L. Ding, W. W. Y. Ng, P. P. K. Chan, D. L. Wu, X. L. Chen, D S. Yeung, "RFID indoor positioning using RBFNN with L-GEM," In the Proceedings of IEEE 2010 International Conference on Machine Learning and Cybernetics, Qingdao, China, pp. 1147-1152, 2010.

[19] A. K. M. M. Hossain, W. S. Soh, "A comprehensive study of Bluetooth signal parameters for localization," In the Proceedings of 18th Annual IEEE International Symposium on Personal, Indoor and Mobile Radio Communications (PIMRC'07), Athens, Greece, pp. 1-5, 2007.

[20] F. Subhan, H. Hasbullah, A. Rozyyev, S. T. Bakhsh, "Indoor positioning in Bluetooth networks using fingerprinting and lateration approach," In the Proceedings of 2011 International Conference on Information Science and Applications (ICISA), Jeju Island, Korea, pp. 1-9, 2001.

[21] W. P. Chen, X. F. Meng, "A cooperative localization scheme for Zigbee-based wireless sensor networks," In the Proceedings of 14th IEEE International Conference on Networks, Singapore, pp. 1-5, 2006.

[22] G. Goncalo, S. Helena, "Indoor location system using ZigBee technology," In the Proceedings of Third International Conference on Sensor Technologies and Applications, Athens/Glyfada, Greece, pp. 152-157, 2009.

[23] S. Merat, W. Almuhtadi, "Wireless network channel quality estimation inside reactor building using RSSI measurement of wireless sensor network," In the Proceedings of Canadian Conference on Electrical and Computer Engineering, Calgary, AB, Canada, pp. 339-341, 2009. 
[24] H. C. Chen, Y. J. Chen, C. Y. Chen, S. M. T. Wang, J. P. Yang, R. C. Hwang, "A new indoor positioning technique based on neural network," Advanced Science Letters, vol. 19, no. 7, pp. 2029-2033, 2013.

[25] A. Zaknich, "Introduction to the modified probabilistic neural network for general signal processing applications," IEEE Transactions on Signal Processing, vol. 46, no. 7, pp. 1980-1990, 1998.

[26] R. C. Chen, Y. H. Lin, "Using ZigBee sensor network with artificial neural network for indoor location," In the Proceedings of Eighth International Conference on Natural Computation, Chongqing, China, pp. 290-294, 2012.

[27] M. Altini, D. Brunelli, E. Farella, L. Benini, "Bluetooth indoor localization with multiple neural networks," In the Proceedings of 5th IEEE International Symposium on Wireless Pervasive Computing (ISWPC), Modena, Italy, pp. 295-300, 2010.

[28] Y. S. Lin, R. C. Chen, Y. C. Lin, "An indoor location identification system based on neural network and genetic algorithm," In the Proceedings of 3rd International Conference on Awareness Science and Technology (iCAST), Dalian, China, pp. 193-198, 2011.

[29] H. Mohammad, A. F. Ozan, A. N. Ali, P. Aveh, "Neural network assisted identification of the absence of direct path in indoor localization," In the Proceedings of IEEE Global Telecommunications Conference, Washington, DC, USA, pp. 387-392, 2007.

[30] S. H. Fang, T. N. Lin, "Indoor location system based on discriminant-adaptive neural network in IEEE 802.11 environments," IEEE Transactions on Neural Networks, vol. 19, no. 11, pp. 1973-1978, 2008.
[31] C. Laoudias, D. G. Eliades, P. Kemppi, C. G. Panayiotou, M. M Polycarpou, "Indoor localization using neural networks with location fingerprints," Lecture Notes in Computer Science 19th International Conference on Artificial Neural Networks, Limassol, Cyprus, vol. 5769, no. 2, pp. 954-963, 2009.

[32] A. Cidronali, S. Maddio, G. Giorgetti, G. Manes, "Analysis and performance of a smart antenna for $2.45-\mathrm{GHz}$ single-anchor indoor positioning," IEEE Trans. Microw. Theory Tech., vol. 58, no. 1, pp. 21-31, 2010.

[33] C. H. Lim, Y. Wan, B. P. Ng, C. M. S. See, "A real-time indoor WiFi localization system utilizing smart antennas," IEEE Transactions on Consumer Electronics, vol. 53, no. 2, pp. 618-622, 2007.

[34] X. M. Qing, Z. N. Chen, T. S. P. See, "Sectored antenna array for indoor mono-station UWB positioning applications," In the Proceedings of 3rd European Conference on Antennas and Propagation, Berlin, Germany, pp. 822-825, 2009.

[35] Antennas and Propagation. http://www.radio-electronics.com/info/antennas/yagi/yagi.php

[36] A. Khotanzad, R. C. Hwang, A. Abaye, D. Maratukulam, "An adaptive modular artificial neural network: Hourly load forecaster and its implementation at electric utilities," IEEE Transactions on Power Systems, vol. 10, pp. 1716-1722, 1995.

[37] R. C. Hwang, P. T. Hsu, J. Cheng, C. Y. Chen, C. Y. Chang, H. C. Huang, "The indoor positioning technique based on neural networks," In the Proceedings of IEEE International Conference on Signal Processing, Communications and Computing (ICSPCC 2011), Xi'an, China, pp. 225-228, 2011. 